\title{
Extended Cubic Method and Its Convergence Analysis for Generalized Equations
}

\author{
Mohammed Harunor Rashid ${ }^{1 *}$, Md. Zulfikar Ali $^{1}$ and Alain Piétrus ${ }^{2}$ \\ ${ }^{1}$ Department of Mathematics, Faculty of Science, University of Rajshahi, Rajshahi-6205, Bangladesh \\ ${ }^{2}$ Département de Mathématiques, et Informatique, Université des Antilles et de la Guyane, Campus de Fouillole, \\ F-97159 Pointe-à-Pitre, France. \\ Email: harun_math@ru.ac.bd
}

\begin{abstract}
Let $X$ and $Y$ be Banach spaces. Let $\Omega$ be an open subset of $X$. Suppose that $f: X \rightarrow Y$ is Fréchet differentiable in $\Omega$ and $\mathcal{F}: X \rightrightarrows 2^{Y}$ is set-valued mapping with closed graph. In the present paper, for solving the generalized equations $0 \in f(x)+\mathcal{F}(x)$, an extended cubic method $(\mathrm{ECM})$ is introduced and studied its convergence analysis. Indeed, we analyze semi-local and local convergence of the ECM.
\end{abstract}

Keywords: Generalized equations, set-valued mappings, Lipschitz-like mappings, cubic method, semi-local convergence.

\section{Introduction}

Let $X$ and $Y$ be Banach spaces and $\Omega \neq \emptyset$ be an open subset of $X$. Let $f: X \rightarrow Y$ be a Fréchet differentiable function on $\Omega$. Assume that the first and second Fréchet derivatives of $f$ are denoted by $\nabla f$ and $\nabla^{2} f$ respectively. Let $\mathcal{F}$ be a set-valued mapping with closed graph acting between Banach space $X$ and the subsets of $Y$. In this communication, we are interested to approximate the solution of the following generalized equation problem

$$
0 \in f(x)+\mathcal{F}(x)
$$

The inclusion type (1), introduced by Robinson [1,2] as a general tool for describing, analyzing, and solving different problems in a unified manner, has been studied extensively.

Let us recall that the inclusion (1) is an abstract model for various problems:

- when $\mathcal{F}=\{0\},(1)$ is an equation.

- when $\mathcal{F}$ is the positive orthant in $\mathbb{R}^{n},(1)$ is a system of inequalities.

- when $\mathcal{F}$ is the normal cone to a convex and closed set in $X,(1)$ reduces to variational inequalities.

- When $\mathcal{F}=\partial \psi_{C}$ is the subdifferential of the function

$$
\psi_{C}(x)= \begin{cases}0, & \text { if } x \in C \\ +\infty, & \text { otherwise }\end{cases}
$$

(1) is reduced to some minimization problems which has been studied by Robinson [3].

To solve (1), Dontchev [4] introduced the following classical Newton-type method, for each $k=0,1, \ldots$,

$$
0 \in f\left(x_{k}\right)+\nabla f\left(x_{k}\right)\left(x_{k+1}-x_{k}\right)+\mathcal{F}\left(x_{k+1}\right)
$$

under the assumptions the set-valued mapping is pseudo-Lipschitz and the Fréchet derivative of $f$ is Lipschitz on a neighborhood of the solution of (1) and established a quadratic convergence of the method. In his subsequent paper [5], he proved the uniform convergence of the method. By following Dontchev's method, Piétrus [6] obtained a super-linear convergence when the Fréchet derivative of $f$ is Hölder continuous on a neighborhood of the solution of (1) and later he [7] established the uniform convergence of this method in this mild differentiability context. 
Let $x \in X$. By $\mathcal{D}(x)$, we symbolize the subset of $X$ which is defined by

$$
\mathcal{D}(x):=\left\{d \in X: 0 \in f(x)+\nabla f(x) d+\frac{1}{2} \nabla^{2} f(x) d^{2}+\mathcal{F}(x+d)\right\} .
$$

For finding an approximate solution of (1), the extension of Dontchev's indigenous work [8] was done by Geoffroy et al. [9]. Thus, we recall the following cubic method defined by Algorithm 1 which is introduced in $[9]$ :

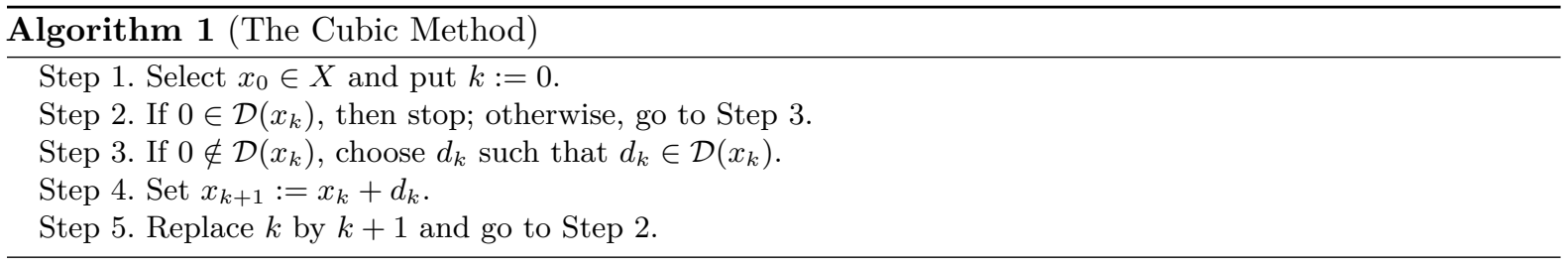

It is remarked that under some suitable conditions around a solution $x^{*}$ of the generalized equation (1), the authors [9, Theorem 3.1] proved that there exists a neighborhood $\Omega$ of $x^{*}$ such that, for any point in $\Omega$, there exists a sequence generated by Algorithm 1 which is cubically convergent to the solution $x^{*}$. This indicates that the convergence result, established in [9], guarantees the existence of a convergent sequence. Therefore, for any initial point near to a solution, the sequences generated by Algorithm 1 are not uniquely defined and not every generated sequence is convergent. Hence, in view of numerical computation, this kind of method is not convenient in practical application. This drawback motivates us to propose a method 'so-called' extended cubic method defined by Algorithm 2.

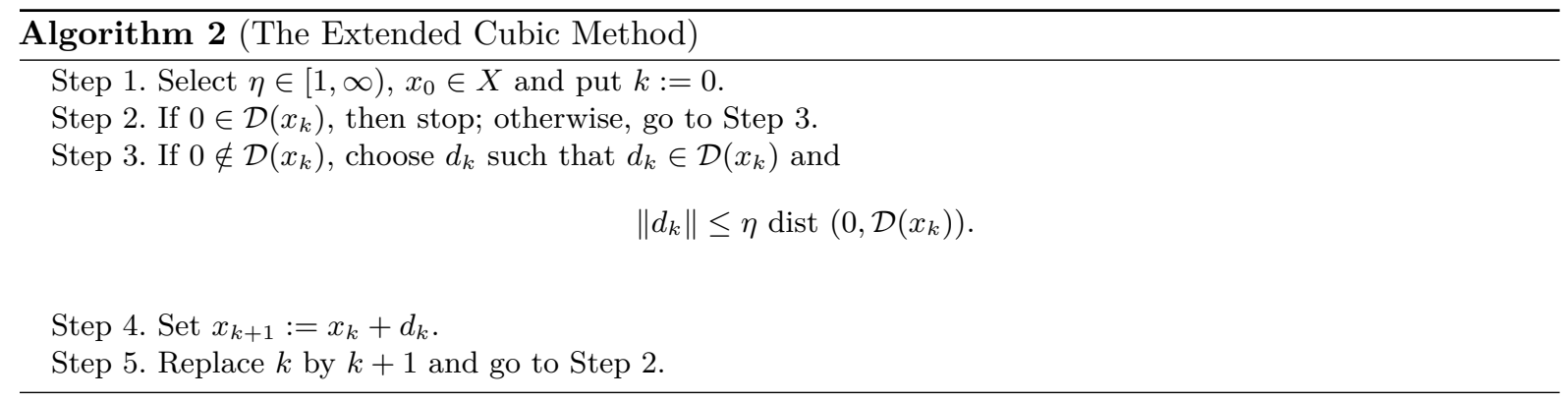

The distinction between Algorithms 1 and 2 is that Algorithm 2 generates at least one sequence and every generated sequence is convergent but this does not happen for Algorithm 1. Since the sequences generated by Algorithm 1 are not uniquely defined, in comparison with Algorithms 1 and 2, we can infer that Algorithm 2 is more precise than Algorithm 1 in numerical computation.

It is also remarked that if we replace the set $\mathcal{D}(x)$ by

$$
\mathcal{D}(x):=\{d \in X: 0 \in f(x)+\nabla f(x) d+\mathcal{F}(x+d)\}
$$

the Algorithm 2 introduced in the present paper will be the same with the Algorithm given in [10]. There have a lot of fruitful works on semilocal analysis for solving (1); see for example $[11,12,13,14,15,16]$.

Rashid et al. [10] established semilocal convergence analysis for solving the generalized equation problem (1), which was the extension of Dontchev's work in [4]. Rashid [17] introduced a variant of Newton-type Method for solving (1) and obtained its semilocal and local convergence results. The same author [18] associated extended Newton-type method for solving a variational inclusion of the form

$$
0 \in f(x)+g(x)+\mathcal{F}(x),
$$


where $g: X \rightarrow Y$ admits first order divided difference and established its semilocal and local convergence results for solving (1). As far we know, there doesn't have any other study on semilocal analysis for the Algorithm 1.

The aim of this study is to analyze the semilocal convergence for the extended cubic method defined by Algorithm 2. More clearly, when $\nabla^{2} f$ is continuous and the set-valued mapping $(f+F)^{-1}$ is Lipschitz-like, the sequence generated by Algorithm 2 converges quadratically to the solution of (1) where as the sequence generated by Algorithm 2 converges cubically to the solution of (1) if $\nabla^{2} f$ is Lipschitz continuous and the set-valued mapping $(f+F)^{-1}$ is Lipschitz-like.

The main results are the convergence criteria, established in Sect.3, which, based on the information around the initial point, provides some sufficient conditions ensuring the convergence to a solution of any sequence generated by Algorithm 2. As a consequence, local convergence results for the cubic method are obtained.

This paper is organized as follows: In Section 2, we recall some necessary notations, notions and some preliminary results. In Section 3, we consider the extended cubic method for solving the generalized equation as well as using the concept of Lipchitz-like mappings, we establish existence results of solutions of the generalized equation and convergence results of the extended cubic method. In the last section, we give a summary of the major results presented in this paper.

\section{Definitions and Preliminary Results}

Assume that $X$ and $Y$ are two real or complex Banach spaces. Let $x \in X$ and $\mathbb{B}(x, r)=\{y:\|y-x\| \leq r\}$ denote the closed ball centered at $x$ with radius $r>0$. Let $\Gamma: X \rightrightarrows 2^{Y}$ be a set-valued mapping. The domain of $\Gamma$, denoted by $\operatorname{dom} \Gamma$, is defined by

$$
\operatorname{dom} \Gamma:=\{x \in X: \Gamma(x) \neq \emptyset\} .
$$

The inverse and the graph of $\Gamma$, denoted by $\Gamma^{-1}$ and gph $\Gamma$ respectively, are defined by

$$
\Gamma^{-1}(y):=\{x \in X: y \in \Gamma(x)\} \quad \text { for each } y \in Y
$$

and

$$
\operatorname{gph} \Gamma:=\{(x, y) \in X \times Y: y \in \Gamma(x)\} .
$$

Let $B \subseteq X$. The distance from a point $x \in X$ to a set $B$ is defined by

$$
\operatorname{dist}(x, B):=\inf _{b \in B}\|x-b\|
$$

and the excess from the set $A$ to the set $B \subseteq X$ is defined by

$$
e(B, A)=\sup _{x \in B}\{\operatorname{dist}(x, A)\}
$$

The notions of pseudo-Lipschitz and Lipchitz-like set-valued mappings are due to [10]. Aubin [19,20] introduced these notions and studied extensively.

Definition 1. Let $G: Y \rightrightarrows 2^{X}$ be a set-valued mapping and let $(\bar{y}, \bar{x}) \in \operatorname{gph} G$. Let $r_{\bar{x}}>0, r_{\bar{y}}>0$ and $M>0$. Then the mapping $G$ is said to be

(a) Lipchitz-like on $\mathbb{B}\left(\bar{y}, r_{\bar{y}}\right)$ relative to $\mathbb{B}\left(\bar{x}, r_{\bar{x}}\right)$ with constant $M$ if the following inequality holds:

$$
e\left(G\left(y_{1}\right) \cap \mathbb{B}\left(\bar{x}, r_{\bar{x}}\right), G\left(y_{2}\right)\right) \leq M\left\|y_{1}-y_{2}\right\| \quad \text { for any } y_{1}, y_{2} \in \mathbb{B}\left(\bar{y}, r_{\bar{y}}\right) .
$$

(b) pseudo-Lipschitz around $(\bar{y}, \bar{x})$ if there exist constants $a>0, b>0$ and $M^{\prime}>0$ such that $G$ is Lipchitz-like on $\mathbb{B}(\bar{y}, b)$ relative to $\mathbb{B}(\bar{x}, a)$ with constant $M^{\prime}$.

The following lemma has taken from [10]. This lemma employs a vital role for proving the convergence analysis. 
Lemma 1. Let $G: Y \rightrightarrows 2^{X}$ be a set-valued mapping and let $(\bar{y}, \bar{x}) \in \operatorname{gph} G$. Assume that $G$ is Lipschitzlike on $\mathbb{B}\left(\bar{y}, r_{\bar{y}}\right)$ relative to $\mathbb{B}\left(\bar{x}, r_{\bar{x}}\right)$ with constant $M$. Then

$$
\operatorname{dist}(x, G(y)) \leq M \operatorname{dist}\left(y, G^{-1}(x)\right)
$$

holds for every $x \in \mathbb{B}\left(\bar{x}, r_{\bar{x}}\right)$ and $y \in \mathbb{B}\left(\bar{y}, \frac{r_{\bar{y}}}{3}\right)$ satisfying $\operatorname{dist}\left(y, G^{-1}(x)\right) \leq \frac{r_{\bar{y}}}{3}$.

We would like to finish this section with the following lemma that is known in [21].

Lemma 2. Let $\Phi: X \rightrightarrows 2^{X}$ be a set-valued mapping. Let $\bar{x} \in X, c>0$ and $0<r<1$ be such that

$$
\operatorname{dist}(\bar{x}, \Phi(\bar{x}))<c(1-r)
$$

and

$$
e\left(\Phi\left(x_{1}\right) \cap \mathbb{B}(\bar{x}, c), \Phi\left(x_{2}\right)\right) \leq r\left\|x_{1}-x_{2}\right\| \quad \text { for any } x_{1}, x_{2} \in \mathbb{B}(\bar{x}, c) .
$$

Then $\Phi$ has a fixed point in $\mathbb{B}(\bar{x}, c)$, that is, there exists $x \in \mathbb{B}(\bar{x}, c)$ such that $x \in \Phi(x)$. Moreover, if $\Phi$ is single-valued, then the fixed point of $\Phi$ in $\mathbb{B}(\bar{x}, c)$ is unique.

\section{Convergence Analysis of Extended Cubic Method}

This section is devoted to prove the existence and convergence of the sequences generated by the extended cubic method defined by Algorithm 2. To this end, let $x \in X$ and let us define the mapping $T_{x}$ by

$$
T_{x}(\cdot):=f(x)+\nabla f(x)(\cdot-x)+\frac{1}{2} \nabla^{2} f(x)(\cdot-x)^{2}+\mathcal{F}(\cdot) .
$$

Then for the construction of $\mathcal{D}(x)$, we have that

$$
\mathcal{D}(x)=\left\{d \in X: 0 \in T_{x}(x+d)\right\}=\left\{d \in X: x+d \in T_{x}{ }^{-1}(0)\right\} .
$$

Moreover, for any $v \in X$ and $y \in Y$, the inclusions

$$
v \in T_{x}^{-1}(y) \text { and } y \in f(x)+\nabla f(x)(v-x)+\frac{1}{2} \nabla^{2} f(x)(v-x)^{2}+\mathcal{F}(v) .
$$

are equivalent. In particular,

$$
\bar{x} \in T_{\bar{x}}^{-1}(\bar{y}) \quad \text { for each }(\bar{x}, \bar{y}) \in \operatorname{gph}(f+\mathcal{F}) .
$$

The following result is due to [22]. This result establishes the equivalence relation between $(f+\mathcal{F})^{-1}$ and $T_{\bar{x}}^{-1}$.

Lemma 3. Let $f: X \rightarrow Y$ be a function and let $(\bar{x}, \bar{y}) \in \operatorname{gph}(f+\mathcal{F})$. Assume that $f$ is twice differentiable in an open neighborhood $\Omega$ of $\bar{x}$ and that its second-order derivative is continuous at $\bar{x}$. Then the following are equivalent:

(i) The mapping $(f+\mathcal{F})^{-1}$ is pseudo-Lipschitz at $(\bar{y}, \bar{x})$;

(ii) The mapping $T_{\bar{x}}^{-1}(\cdot)$ is pseudo-Lipschitz at $(\bar{y}, \bar{x})$.

Let $r_{\bar{x}}>0, r_{\bar{y}}>0$ and $(\bar{x}, \bar{y}) \in \operatorname{gph}(f+\mathcal{F})$. Then, the closed graph property of the set-valued mapping $f+\mathcal{F}$ implies that $f+\mathcal{F}$ is continuous at $\bar{x}$ for $\bar{y}$, that is,

$$
\lim _{x \rightarrow \bar{x}} \operatorname{dist}(\bar{y}, f(x)+\mathcal{F}(x))=0 .
$$

Assume that $\mathbb{B}\left(\bar{x}, r_{\bar{x}}\right) \subseteq \Omega \cap \operatorname{dom} \mathcal{F}$. Moreover, by Lemma 3 we assume that the mapping $T_{\bar{x}}^{-1}$ is Lipschitz-like on $\mathbb{B}\left(\bar{y}, r_{\bar{y}}\right)$ relative to $\mathbb{B}\left(\bar{x}, r_{\bar{x}}\right)$ with constant $M$, that is,

$$
e\left(T_{\bar{x}}^{-1}\left(y_{1}\right) \cap \mathbb{B}\left(\bar{x}, r_{\bar{x}}\right), T_{\bar{x}}^{-1}\left(y_{2}\right)\right) \leq M\left\|y_{1}-y_{2}\right\| \forall y_{1}, y_{2} \in \mathbb{B}\left(\bar{y}, r_{\bar{y}}\right) .
$$


Let $\varepsilon>0$ and set

$$
\alpha:=\min \left\{r_{\bar{y}}-\frac{13}{4} \varepsilon r_{\bar{x}}^{2}, \frac{r_{\bar{x}}(2-5 M \varepsilon)}{20 M}\right\} .
$$

Then

$$
\alpha>0 \text { if and only if } \varepsilon<\min \left\{\frac{4 r_{\bar{y}}}{13 r_{\bar{x}}^{2}}, \frac{2}{5 M}\right\} .
$$

The following lemma plays a principal role for convergence analysis of the extended cubic method. The proof is a refinement of the one for [10, Lemma 3.1].

Lemma 4. Let $T_{\bar{x}}^{-1}$ be a Lipschitz-like mapping on $\mathbb{B}\left(\bar{y}, r_{\bar{y}}\right)$ relative to $\mathbb{B}\left(\bar{x}, r_{\bar{x}}\right)$ with constant $M$. Let $x \in \mathbb{B}\left(\bar{x}, \frac{r_{\bar{x}}}{2}\right)$. Assume that $\nabla f$ and $\nabla^{2} f$ are continuous at $\bar{x}$ on $\mathbb{B}\left(\bar{x}, \frac{r_{\bar{x}}}{2}\right)$ with the same constant $\varepsilon$ defined by (10). Let $\alpha$ be defined in (9) so that (10) is satisfied. Then the mapping $T_{x}^{-1}$ is Lipschitz-like on $\mathbb{B}(\bar{y}, \alpha)$ relative to $\mathbb{B}\left(\bar{x}, \frac{r_{\bar{x}}}{2}\right)$ with constant $\frac{5 M}{2-5 M \varepsilon}$ i.e.

$$
e\left(T_{x}^{-1}\left(t_{1}\right) \cap \mathbb{B}\left(\bar{x}, \frac{r_{\bar{x}}}{2}\right), T_{x}^{-1}\left(t_{2}\right)\right) \leq \frac{5 M}{2-5 M \varepsilon}\left\|t_{1}-t_{2}\right\| \quad \text { for every } t_{1}, t_{2} \in \mathbb{B}(\bar{y}, \alpha) .
$$

Proof. Since $\alpha$ is defined in (9) so that (10) is satisfied, then it is clear that $\alpha>0$. Now let

$$
t_{1}, t_{2} \in \mathbb{B}(\bar{y}, \alpha) \quad \text { and } \quad u^{\prime} \in T_{x}^{-1}\left(t_{1}\right) \cap \mathbb{B}\left(\bar{x}, \frac{r_{\bar{x}}}{2}\right) .
$$

To complete the proof, it is sufficient to show that there exists $u^{\prime \prime} \in T_{x}^{-1}\left(t_{2}\right)$ such that

$$
\left\|u^{\prime}-u^{\prime \prime}\right\| \leq \frac{5 M}{2-5 M \varepsilon}\left\|t_{1}-t_{2}\right\| .
$$

To finish this, we need to verify that there exists a sequence $\left\{x_{k}\right\} \subseteq \mathbb{B}\left(\bar{x}, r_{\bar{x}}\right)$ such that

$$
\begin{aligned}
& t_{2} \in f(x)+\nabla f(x)\left(x_{k-1}-x\right)+\nabla f(\bar{x})\left(x_{k}-x_{k-1}\right)+\frac{1}{2} \nabla^{2} f(x)\left(x_{k-1}-x\right)^{2} \\
& +\frac{1}{2} \nabla^{2} f(\bar{x})\left(\left(x_{k}-\bar{x}\right)^{2}-\left(x_{k-1}-\bar{x}\right)^{2}\right)+\mathcal{F}\left(x_{k}\right) . \\
& \quad \text { and } \quad\left\|x_{k}-x_{k-1}\right\| \leq \frac{5 M}{2}\left\|t_{1}-t_{2}\right\|\left(\frac{5 M}{2} \varepsilon\right)^{k-2}
\end{aligned}
$$

hold for each $k=2,3,4, \ldots$ We proceed by induction on $k$. Write

$$
\begin{aligned}
a_{i}:= & t_{i}-f(x)-\nabla f(x)\left(x^{\prime}-x\right)-\frac{1}{2} \nabla^{2} f(x)\left(x^{\prime}-x\right)^{2}+f(\bar{x})+\nabla f(\bar{x})\left(x^{\prime}-\bar{x}\right) \\
& +\frac{1}{2} \nabla^{2} f(\bar{x})\left(x^{\prime}-\bar{x}\right)^{2} \quad \text { for each } i=1,2 .
\end{aligned}
$$

Note by (11) that

$$
\left\|x-x^{\prime}\right\| \leq\|x-\bar{x}\|+\left\|\bar{x}-x^{\prime}\right\| \leq r_{\bar{x}} .
$$

Furthermore, we have, for (14), that

$$
\begin{aligned}
\left\|a_{i}-\bar{y}\right\| \leq & \left\|t_{i}-\bar{y}\right\|+\left\|f\left(x^{\prime}\right)-f(x)-\nabla f(x)\left(x^{\prime}-x\right)-\frac{1}{2} \nabla^{2} f(x)\left(x^{\prime}-x\right)^{2}\right\|+\| f\left(x^{\prime}\right)-f(\bar{x}) \\
& -\nabla f(\bar{x})\left(x^{\prime}-\bar{x}\right)-\frac{1}{2} \nabla^{2} f(\bar{x})\left(x^{\prime}-\bar{x}\right)^{2} \| .
\end{aligned}
$$

Since $\nabla f$ and $\nabla^{2} f$ are continuous at $\bar{x}$ with the same constant $\varepsilon>0$, we have that

$$
\begin{aligned}
\|f(x)-f(\bar{x})-\nabla f(\bar{x})(x-\bar{x})\| & =\left\|\int_{0}^{1}[\nabla f(\bar{x}+t(x-\bar{x}))-\nabla f(\bar{x})](x-\bar{x}) d t\right\| \\
& \leq \int_{0}^{1}\|\nabla f(\bar{x}+t(x-\bar{x}))-\nabla f(\bar{x})\|\|x-\bar{x}\| d t \\
& \leq \varepsilon\|x-\bar{x}\| \int_{0}^{1} d t=\varepsilon\|x-\bar{x}\|,
\end{aligned}
$$


and

$$
\begin{aligned}
& \left\|f(x)-f(\bar{x})-\nabla f(\bar{x})(x-\bar{x})-\frac{1}{2} \nabla^{2} f(\bar{x})(x-\bar{x})^{2}\right\| \\
= & \left\|\int_{0}^{1}\left[\nabla f(\bar{x}+t(x-\bar{x}))-\nabla f(\bar{x})-\nabla^{2} f(\bar{x})(\bar{x}+t(x-\bar{x})-\bar{x})\right](x-\bar{x}) d t\right\| \\
\leq & \int_{0}^{1}\left\|\nabla f(\bar{x}+t(x-\bar{x}))-\nabla f(\bar{x})-\nabla^{2} f(\bar{x})(\bar{x}+t(x-\bar{x})-\bar{x})\right\|\|x-\bar{x}\| d t \\
\leq & \varepsilon\|x-\bar{x}\|^{2} \int_{0}^{1} d t=\varepsilon\|x-\bar{x}\|^{2} .
\end{aligned}
$$

Then from (16), using the relations in (11), (15) and the relation $\bar{r} \leq r_{\bar{y}}-\frac{13}{4} \varepsilon{r_{\bar{x}}}^{2}$ by (9), we have that

$$
\begin{aligned}
\left\|a_{i}-\bar{y}\right\| & \leq \alpha+\varepsilon\left(\left\|x^{\prime}-x\right\|^{2}+\left\|x^{\prime}-\bar{x}\right\|^{2}\right) \leq \bar{r}+\varepsilon\left(r_{\bar{x}}^{2}+\frac{r_{\bar{x}}^{2}}{4}\right) \\
& =\alpha+\frac{5}{4} \varepsilon r_{\bar{x}}{ }^{2} \leq r_{\bar{y}} .
\end{aligned}
$$

That is $a_{i} \in \mathbb{B}\left(\bar{y}, r_{\bar{y}}\right)$ for each $i=1,2$. Define $x_{1}:=u^{\prime}$. Then $x_{1} \in T_{x}^{-1}\left(t_{1}\right)$ by (11) and it follows from (5) that

$$
t_{1} \in f(x)+\nabla f(x)\left(x_{1}-x\right)+\frac{1}{2} \nabla^{2} f(x)\left(x_{1}-x\right)^{2}+\mathcal{F}\left(x_{1}\right) .
$$

This can be written in another form as follows:

$$
\begin{aligned}
& \left.t_{1}+f(\bar{x})+\nabla f(\bar{x})\left(x_{1}-\bar{x}\right)\right)+\frac{1}{2} \nabla^{2} f(\bar{x})\left(x_{1}-\bar{x}\right)^{2} \in f(x)+\nabla f(x)\left(x_{1}-x\right)+\frac{1}{2} \nabla^{2} f(x)\left(x_{1}-x\right)^{2} \\
& +\mathcal{F}\left(x_{1}\right)+f(\bar{x})+\nabla f(\bar{x})\left(x_{1}-\bar{x}\right)+\frac{1}{2} \nabla^{2} f(\bar{x})\left(x_{1}-\bar{x}\right)^{2} .
\end{aligned}
$$

This, by the definition of $a_{1}$, implies that

$$
a_{1} \in f(\bar{x})+\nabla f(\bar{x})\left(x_{1}-\bar{x}\right)++\frac{1}{2} \nabla^{2} f(\bar{x})\left(x_{1}-\bar{x}\right)^{2}+\mathcal{F}\left(x_{1}\right) .
$$

Hence $x_{1} \in T_{\bar{x}}^{-1}\left(a_{1}\right)$ by (5). This together with (11) implies that

$$
x_{1} \in T_{\bar{x}}^{-1}\left(a_{1}\right) \cap \mathbb{B}\left(\bar{x}, r_{\bar{x}}\right) .
$$

By the assumed Lipschitz-like property of $T_{\bar{x}}^{-1}$ and noting that $a_{1}, a_{2} \in \mathbb{B}\left(\bar{y}, r_{\bar{y}}\right)$, it follows from (8) that there exists $x_{2} \in T_{\bar{x}}^{-1}\left(a_{2}\right)$ such that

$$
\left\|x_{2}-x_{1}\right\| \leq M\left\|a_{1}-a_{2}\right\|=M\left\|t_{1}-t_{2}\right\|<\frac{5 M}{2}\left\|t_{1}-t_{2}\right\| .
$$

Moreover, by the construction of $t_{2}$ and noting $x_{1}=u^{\prime}$, we have

$$
\begin{aligned}
x_{2} \in T_{\bar{x}}^{-1}\left(a_{2}\right)= & T_{\bar{x}}^{-1}\left(t_{2}-f(x)-\nabla f(x)\left(x_{1}-x\right)-\frac{1}{2} \nabla^{2} f(x)\left(x_{1}-x\right)^{2}\right. \\
& \left.+f(\bar{x})+\nabla f(\bar{x})\left(x_{1}-\bar{x}\right)+\frac{1}{2} \nabla^{2} f(\bar{x})\left(x_{1}-\bar{x}\right)^{2}\right),
\end{aligned}
$$

which, together with (5), implies that

$$
\begin{aligned}
t_{2} \in & f(x)+\nabla f(x)\left(x_{1}-x\right)+\nabla f(\bar{x})\left(x_{2}-x_{1}\right)+\frac{1}{2} \nabla^{2} f(x)\left(x_{1}-x\right)^{2} \\
& +\frac{1}{2} \nabla^{2} f(\bar{x})\left(\left(x_{2}-\bar{x}\right)^{2}-\left(x_{1}-\bar{x}\right)^{2}\right)+\mathcal{F}\left(x_{2}\right) .
\end{aligned}
$$

This shows that (12) and (13) hold with generated points $x_{1}, x_{2}$. 
Assume that $x_{1}, x_{2}, \ldots, x_{n}$ are obtained so that (12) and (13) hold for $k=2,3, \ldots, n$. We need to construct $x_{n+1}$ such that (12) and (13) are also true for $k=n+1$. For this purpose, set

$$
\begin{aligned}
a_{i}^{n}:= & t_{2}-f(x)-\nabla f(x)\left(x_{n+i-1}-x\right)-\frac{1}{2} \nabla^{2} f(x)\left(x_{n+i-1}-x\right)^{2}+f(\bar{x}) \\
& +\nabla f(\bar{x})\left(x_{n+i-1}-\bar{x}\right)+\frac{1}{2} \nabla^{2} f(\bar{x})\left(x_{n+i-1}-\bar{x}\right)^{2} \quad \text { for each } i=0,1
\end{aligned}
$$

Then, for $i=0,1$, we obtain that

$$
\begin{aligned}
& \left\|a_{0}^{n}-a_{1}^{n}\right\| \\
= & \|(\nabla f(x)-\nabla f(\bar{x}))\left(x_{n}-x_{n-1}\right)+\frac{1}{2} \nabla^{2} f(x)\left(\left(x_{n}-x\right)^{2}-\left(x_{n-1}-x\right)^{2}\right) \\
& -\frac{1}{2} \nabla^{2} f(\bar{x})\left(\left(x_{n}-\bar{x}\right)^{2}-\left(x_{n-1}-\bar{x}\right)^{2}\right) \| \\
= & \|(\nabla f(x)-\nabla f(\bar{x}))\left(x_{n}-x_{n-1}\right)+\frac{1}{2} \nabla^{2} f(x)\left(\left(x_{n}-x_{n-1}+x_{n-1}-x\right)^{2}\right. \\
& \left.-\left(x_{n-1}-x\right)^{2}\right)-\frac{1}{2} \nabla^{2} f(\bar{x})\left(\left(x_{n}-x_{n-1}+x_{n-1}-\bar{x}\right)^{2}-\left(x_{n-1}-\bar{x}\right)^{2}\right) \| \\
\leq & \|\nabla f(x)-\nabla f(\bar{x})\|\left\|x_{n}-x_{n-1}\right\|+\frac{1}{2}\left\|\nabla^{2} f(x)-\nabla^{2} f(\bar{x})\right\|\left\|x_{n}-x_{n-1}\right\|^{2} \\
& +\left\|\nabla^{2} f(x)\left(x_{n-1}-x\right)-\nabla^{2} f(\bar{x})\left(x_{n-1}-\bar{x}\right)\right\|\left\|x_{n}-x_{n-1}\right\| .
\end{aligned}
$$

For all $z \in \mathbb{B}\left(\bar{x}, \frac{r_{\bar{x}}}{2}\right), x \mapsto \nabla f(x), x \mapsto \nabla^{2} f(x)$ and $x \mapsto \nabla^{2} f(x)(z-x)$ are continuous at $\bar{x}$, thus we have that

$$
\begin{aligned}
\left\|a_{0}^{n}-a_{1}^{n}\right\| \leq & \varepsilon\left\|x_{n}-x_{n-1}\right\|+\frac{1}{2} \varepsilon\left\|x_{n}-x_{n-1}\right\|^{2}+\varepsilon\|\| x_{n}-x_{n-1} \| \\
= & \varepsilon\left(2\left\|x_{n}-x_{n-1}\right\|+\frac{1}{2}\left\|x_{n}-x_{n-1}\right\|^{2}\right) \\
\leq & \varepsilon\left(2\left\|x_{n}-x_{n-1}\right\|+\frac{1}{2}\left\|x_{n}-x_{n-1}\right\|\right), \text { if the ball } \\
& \mathbb{B}\left(\bar{x}, \frac{r_{\bar{x}}}{2}\right) \text { is sufficiently small } \\
= & \frac{5}{2} \varepsilon\left\|x_{n}-x_{n-1}\right\| .
\end{aligned}
$$

Since $\left\|x_{1}-\bar{x}\right\| \leq \frac{r_{\bar{x}}}{2}$ by $(11)$ and $\left\|t_{1}-t_{2}\right\| \leq 2 \alpha$ by (11), it follows from (13) that

$$
\begin{aligned}
\left\|x_{n}-\bar{x}\right\| & \leq \sum_{j=2}^{n}\left\|x_{j}-x_{j-1}\right\|+\left\|x_{1}-\bar{x}\right\| \\
& \leq 5 M \alpha \sum_{j=2}^{n}\left(\frac{5 M \varepsilon}{2}\right)^{j-2}+\frac{r_{\bar{x}}}{2} \leq \frac{5 M \alpha}{1-\frac{5 M \varepsilon}{2}}+\frac{r_{\bar{x}}}{2} \\
& =\frac{10 M \alpha}{2-5 M \varepsilon}+\frac{r_{\bar{x}}}{2} .
\end{aligned}
$$

By (9), we have $\alpha \leq \frac{r_{\bar{x}}(2-5 M \varepsilon)}{20 M}$ and so

$$
\left\|x_{n}-\bar{x}\right\| \leq r_{\bar{x}}
$$

Therefore, we obtain that

$$
\left\|x_{n}-x\right\| \leq\left\|x_{n}-\bar{x}\right\|+\|\bar{x}-x\| \leq \frac{3}{2} r_{\bar{x}} .
$$


Furthermore, using (11) and (19), one has that, for each $i=0,1$,

$$
\begin{aligned}
\left\|a_{i}^{n}-\bar{y}\right\| \leq & \left\|t_{i}-\bar{y}\right\|+\left\|f\left(x_{n+i-1}\right)-f(x)-\nabla f(x)\left(x_{n+i-1}-x\right)-\frac{1}{2} \nabla^{2} f(x)\left(x_{n+i-1}-x\right)^{2}\right\| \\
& +\left\|f\left(x_{n+i-1}\right)-f(\bar{x})-\nabla f(\bar{x})\left(x_{n+i-1}-\bar{x}\right)-\frac{1}{2} \nabla^{2} f(\bar{x})\left(x_{n+i-1}-\bar{x}\right)^{2}\right\| \\
\leq & \alpha+\varepsilon\left(\left\|x_{n+i-1}-x\right\|^{2}+\left\|x_{n+i-1}-\bar{x}\right\|^{2}\right) \leq \alpha+\varepsilon\left(\frac{9}{4} r_{\bar{x}}{ }^{2}+r_{\bar{x}}{ }^{2}\right) \\
= & \alpha+\frac{13}{4} \varepsilon r_{\bar{x}}{ }^{2} .
\end{aligned}
$$

It follows, from the definition of $\alpha$ in (9), that $a_{i}^{n} \in \mathbb{B}\left(\bar{y}, r_{\bar{y}}\right)$ for each $i=0,1$. Since assumption (12) holds for $k=n$, we have

$$
\begin{aligned}
t_{2} \in & f(x)+\nabla f(x)\left(x_{n-1}-x\right)+\nabla f(\bar{x})\left(x_{n}-x_{n-1}\right)+\frac{1}{2} \nabla^{2} f(x)\left(x_{n-1}-x\right)^{2} \\
& +\frac{1}{2} \nabla^{2} f(\bar{x})\left[\left(x_{n}-\bar{x}\right)^{2}-\left(x_{n-1}-\bar{x}\right)^{2}\right]+\mathcal{F}\left(x_{n}\right),
\end{aligned}
$$

which can be rewritten as

$$
\begin{aligned}
& \left.t_{2}+f(\bar{x})+\nabla f(\bar{x})\left(x_{n-1}-\bar{x}\right)\right)+\frac{1}{2} \nabla^{2} f(\bar{x})\left(x_{n-1}-\bar{x}\right)^{2} \in f(x)+\nabla f(x)\left(x_{n-1}-x\right) \\
& +\nabla f(\bar{x})\left(x_{n}-x_{n-1}\right)+\frac{1}{2} \nabla^{2} f(x)\left(x_{n-1}-x\right)^{2}+\frac{1}{2} \nabla^{2} f(\bar{x})\left[\left(x_{n}-\bar{x}\right)^{2}-\left(x_{n-1}-\bar{x}\right)^{2}\right]+\mathcal{F}\left(x_{n}\right)+f(\bar{x}) \\
& +\nabla f(\bar{x})\left(x_{n-1}-\bar{x}\right)+\frac{1}{2} \nabla^{2} f(\bar{x})\left(x_{n-1}-\bar{x}\right)^{2} .
\end{aligned}
$$

Then by the definition of $a_{0}^{n}$, we have that $a_{0}^{n} \in f(\bar{x})+\nabla f(\bar{x})\left(x_{n}-\bar{x}\right)+\frac{1}{2} \nabla^{2} f(\bar{x})\left(x_{n}-\bar{x}\right)^{2}+\mathcal{F}\left(x_{n}\right)$. This, together with (5) and (18), yields that

$$
x_{n} \in T_{\bar{x}}^{-1}\left(a_{0}^{n}\right) \cap \mathbb{B}\left(\bar{x}, r_{\bar{x}}\right) .
$$

Using (8) again, there exists an element $x_{n+1} \in T_{\bar{x}}^{-1}\left(a_{1}^{n}\right)$ such that

$$
\left\|x_{n+1}-x_{n}\right\| \leq M\left\|a_{0}^{n}-a_{1}^{n}\right\| \leq \frac{5 M}{2}\left\|t_{1}-t_{2}\right\|\left(\frac{5 M}{2} \varepsilon\right)^{n-1},
$$

where the last inequality holds by (17). By the definition of $a_{1}^{n}$, we have

$$
\begin{aligned}
x_{n+1} \in T_{\bar{x}}^{-1}\left(a_{1}^{n}\right)= & T_{\bar{x}}^{-1}\left(t_{2}-f(x)-\nabla f(x)\left(x_{n}-x\right)-\frac{1}{2} \nabla^{2} f(x)\left(x_{n}-x\right)^{2}\right. \\
& \left.+f(\bar{x})+\nabla f(\bar{x})\left(x_{n}-\bar{x}\right)+\frac{1}{2} \nabla^{2} f(\bar{x})\left(x_{n}-\bar{x}\right)^{2}\right),
\end{aligned}
$$

which, together with (5), implies that

$$
\begin{aligned}
t_{2} \in & f(x)+\nabla f(x)\left(x_{n}-x\right)+\nabla f(\bar{x})\left(x_{n+1}-x_{n}\right)+\frac{1}{2} \nabla^{2} f(x)\left(x_{n}-x\right)^{2} \\
& +\frac{1}{2} \nabla^{2} f(\bar{x})\left(\left(x_{n+1}-\bar{x}\right)^{2}-\left(x_{n}-\bar{x}\right)^{2}\right)+\mathcal{F}\left(x_{n+1}\right) .
\end{aligned}
$$

This, together with (20), completes the induction step and ensure the existence of a sequence $\left\{x_{n}\right\}$ satisfying (12) and (13).

Since $\frac{5 M \varepsilon}{2}<1$, we see from (13) that $\left\{x_{k}\right\}$ is a Cauchy sequence and hence it is convergent, say to $x^{\prime \prime}$, that is $x^{\prime \prime}:=\lim _{k \rightarrow \infty} x_{k}$. Note that $F$ has closed graph. Then, taking limit in (12), we get $t_{2} \in f(x)+\nabla f(x)\left(x^{\prime \prime}-x\right)+\frac{1}{2} \nabla^{2} f(x)\left(x^{\prime \prime}-x\right)^{2}+\mathcal{F}\left(x^{\prime \prime}\right)$ and so $x^{\prime \prime} \in T_{x}^{-1}\left(t_{2}\right)$. Moreover,

$$
\begin{aligned}
\left\|x^{\prime}-x^{\prime \prime}\right\| & \leq \limsup _{n \rightarrow \infty} \sum_{k=2}^{n}\left\|x_{k}-x_{k-1}\right\| \leq \lim _{n \rightarrow \infty} \sum_{k=2}^{n} \frac{5 M}{2}\left\|t_{1}-t_{2}\right\|\left(\frac{5 M}{2} \varepsilon\right)^{k-2} \\
& =\frac{5 M}{2-5 M \varepsilon}\left\|t_{1}-t_{2}\right\| .
\end{aligned}
$$


This completes the proof of the Lemma 4.

Before going to demonstrate our main results, we define, for each $x \in X$, the mapping $J_{x}: X \rightarrow Y$ by

$$
J_{x}(\cdot):=f(\bar{x})+\nabla f(\bar{x})(\cdot-\bar{x})+\frac{1}{2} \nabla^{2} f(\bar{x})(\cdot-\bar{x})^{2}-f(x)-\nabla f(x)(\cdot-x)-\frac{1}{2} \nabla^{2} f(x)(\cdot-x)^{2}
$$

and the set-valued mapping $\Phi_{x}: X \rightrightarrows 2^{X}$ by

$$
\Phi_{x}(\cdot)=T_{\bar{x}}^{-1}\left[J_{x}(\cdot)\right] .
$$

Then, for any $x^{\prime}, x^{\prime \prime} \in X$, we have that

$$
\begin{aligned}
\left\|J_{x}\left(x^{\prime}\right)-J_{x}\left(x^{\prime \prime}\right)\right\|= & \|(\nabla f(\bar{x})-\nabla f(x))\left(x^{\prime}-x^{\prime \prime}\right)+\frac{1}{2} \nabla^{2} f(\bar{x})\left(\left(x^{\prime}-\bar{x}\right)^{2}-\left(x^{\prime \prime}-\bar{x}\right)^{2}\right) \\
& -\frac{1}{2} \nabla^{2} f(x)\left(\left(x^{\prime}-x\right)^{2}-\left(x^{\prime \prime}-x\right)^{2}\right) \| \\
= & \|(\nabla f(\bar{x})-\nabla f(x))\left(x^{\prime}-x^{\prime \prime}\right)+\frac{1}{2} \nabla^{2} f(\bar{x})\left(\left(x^{\prime}-x^{\prime \prime}+x^{\prime \prime}-\bar{x}\right)^{2}-\left(x^{\prime \prime}-\bar{x}\right)^{2}\right) \\
& -\frac{1}{2} \nabla^{2} f(x)\left(\left(x^{\prime}-x^{\prime \prime}+x^{\prime \prime}-x\right)^{2}-\left(x^{\prime \prime}-x\right)^{2}\right) \| \\
\leq & \|\nabla f(\bar{x})-\nabla f(x)\|\left\|x^{\prime}-x^{\prime \prime}\right\|+\frac{1}{2}\left\|\nabla^{2} f(\bar{x})-\nabla^{2} f(x)\right\|\left\|x^{\prime}-x^{\prime \prime}\right\|^{2} \\
& +\left\|\nabla^{2} f(\bar{x})\left(x^{\prime \prime}-\bar{x}\right)-\nabla^{2} f(x)\left(x^{\prime \prime}-x\right)\right\|\left\|x^{\prime}-x^{\prime \prime}\right\| .
\end{aligned}
$$

\subsection{Quadratic Convergence}

This subsection is devoted to study the quadratic convergence of the sequence generated by Algorithm 2 for solving (1) when $\nabla^{2} f$ is continuous. Thus, the first main theorem of this study, which gives some sufficient conditions confirming the convergence of the extended cubic method with starting point $x_{0}$, read as follows:

Theorem 1. Suppose that $\eta>1$ and that $T_{\bar{x}}^{-1}$ is Lipschitz-like on $\mathbb{B}\left(\bar{y}, r_{\bar{y}}\right)$ relative to $\mathbb{B}\left(\bar{x}, r_{\bar{x}}\right)$ with constant $M$. Let $\nabla^{2} f$ be continuous on $\mathbb{B}\left(\bar{x}, \frac{r_{\bar{x}}}{2}\right)$ with constant $\varepsilon$ and let $\alpha$ be defined by (9). Let $\delta>0$ be such that

(a) $\delta \leq \min \left\{\frac{r_{\bar{x}}}{4}, \frac{\alpha}{3 \varepsilon}, \frac{r_{\bar{y}}}{14 \varepsilon}, 1\right\}$; (b) $5 M(1+10 \eta \delta) \varepsilon \leq 2$ and (c) $\|\bar{y}\|<\varepsilon \delta^{2}$.

Suppose that $f+\mathcal{F}$ is continuous at $\bar{x}$ for $\bar{y}$, i.e. (7) holds. Then there exists some $\hat{\delta}>0$ such that any sequence $\left\{x_{n}\right\}$ generated by Algorithm 2 with starting point in $\mathbb{B}(\bar{x}, \hat{\delta})$ converges quadratically to a solution $x^{*}$ of (1).

Proof. Let

$$
t:=\frac{50 \eta M \varepsilon \delta}{2-5 M \varepsilon} .
$$

Then by assumption (b), we obtain that

$$
t \leq 1
$$

Since $f+\mathcal{F}$ is continuous at $\bar{x}$ for $\bar{y}$, by assumption (c), we can choose $\hat{\delta}$ be such that $0<\hat{\delta} \leq \delta$ and

$$
\operatorname{dist}\left(0, f\left(x_{0}\right)+\mathcal{F}\left(x_{0}\right)\right) \leq \varepsilon \delta^{2} \quad \text { for each } x_{0} \in \mathbb{B}(\bar{x}, \hat{\delta})
$$

Let $x_{0} \in \mathbb{B}(\bar{x}, \hat{\delta})$. To complete the proof, we will proceed by mathematical induction and show that Algorithm 2 generates at least one sequence $\left\{x_{n}\right\}$ and any sequence generated by Algorithm 2 satisfies the following assertions:

$$
\left\|x_{n}-\bar{x}\right\| \leq 2 \delta
$$


and

$$
\left\|x_{n+1}-x_{n}\right\| \leq t\left(\frac{1}{10}\right)^{2^{n}} \delta
$$

for each $n=0,1,2, \ldots$ Define

$$
r_{x}:=\frac{25}{3}\left(M \varepsilon\|x-\bar{x}\|^{2}+M\|\bar{y}\|\right) \quad \text { for each } x \in X .
$$

Since $\eta>1$, it follows from assumption (b) that

$$
5 M(1+10 \delta) \varepsilon \leq 5 M(1+10 \eta \delta) \varepsilon \leq 2 .
$$

This yields

$$
5 M \varepsilon \leq 2 \text { and } 50 M \varepsilon \delta \leq 2 .
$$

Thanks to the assumption (c). Combining the second inequality in (29) and assumption (c) we have, from (28), that

$$
r_{x}<\frac{25}{3}\left(5 M \varepsilon \delta^{2}\right) \leq 2 \delta \quad \text { for each } x \in \mathbb{B}(\bar{x}, 2 \delta) .
$$

Furthermore, since $\delta \leq 1$ and $3 \varepsilon \delta \leq \alpha$ in assumption (a), we have that

$$
\|\bar{y}\|<\varepsilon \delta^{2}=\varepsilon \delta \cdot \delta \leq \frac{\alpha}{3} .
$$

It is obvious that (26) is true for $n=0$. Firstly, we need to show that $x_{1}$ exists and (27) holds for $n=0$. To finish this, we have to prove $\mathcal{D}\left(x_{0}\right) \neq \emptyset$ by applying Lemma 2 to the mapping $\Phi:=\Phi_{x_{0}}$. Let us check that both assumptions (2) and (3) of Lemma 2 hold with $c:=r_{x_{0}}$ and $r:=\frac{22}{25}$. By (6), we note that $\bar{x} \in T_{\bar{x}}^{-1}(\bar{y}) \cap \mathbb{B}(\bar{x}, \delta)$. Thus, according to the definition of the excess $e$ and the mapping $\Phi_{x_{0}}$ in (21), we obtain

$$
\operatorname{dist}\left(\bar{x}, \Phi_{x_{0}}(\bar{x})\right) \leq e\left(T_{\bar{x}}^{-1}(\bar{y}) \cap \mathbb{B}(\bar{x}, \delta), \Phi_{x_{0}}(\bar{x})\right) \leq e\left(T_{\bar{x}}^{-1}(\bar{y}) \cap \mathbb{B}\left(\bar{x}, r_{\bar{x}}\right), T_{\bar{x}}^{-1}\left[J_{x_{0}}(\bar{x})\right]\right)
$$

With the help of continuity property of $\nabla^{2} f$, we obtain that

$$
\begin{aligned}
\left\|J_{x_{0}}(x)-\bar{y}\right\|= & \| f(\bar{x})+\nabla f(\bar{x})(x-\bar{x})+\frac{1}{2} \nabla^{2} f(\bar{x})(x-\bar{x})^{2}-f\left(x_{0}\right)-\nabla f\left(x_{0}\right)\left(x-x_{0}\right) \\
& -\frac{1}{2} \nabla^{2} f\left(x_{0}\right)\left(x-x_{0}\right)^{2}-\bar{y} \| \\
\leq & \left\|f(x)-f\left(x_{0}\right)-\nabla f\left(x_{0}\right)\left(x-x_{0}\right)-\frac{1}{2} \nabla^{2} f\left(x_{0}\right)\left(x-x_{0}\right)^{2}\right\| \\
& +\left\|f(x)-f(\bar{x})-\nabla f(\bar{x})(x-\bar{x})+\frac{1}{2} \nabla^{2} f(\bar{x})(x-\bar{x})^{2}\right\|+\|\bar{y}\| \\
\leq & \varepsilon\left(\left\|x-x_{0}\right\|^{2}+\|x-\bar{x}\|^{2}\right)+\|\bar{y}\| .
\end{aligned}
$$

We note here that $\left\|x_{0}-\bar{x}\right\| \leq \hat{\delta} \leq \delta, 14 \delta \varepsilon \leq r_{\bar{y}}$ by assumption (a) and $\|\bar{y}\|<\varepsilon \delta^{2}$ by assumption (c). It follows from (33) that, for each $x \in \mathbb{B}(\bar{x}, 2 \delta)$,

$$
\begin{aligned}
\left\|J_{x_{0}}(x)-\bar{y}\right\| & \leq \varepsilon\left(\left\|x-x_{0}\right\|^{2}+\|x-\bar{x}\|^{2}\right)+\|\bar{y}\| \leq 14 \varepsilon \delta^{2} \\
& \leq 14 \varepsilon \delta \leq r_{\bar{y}} .
\end{aligned}
$$

Thus, for each $x \in \mathbb{B}(\bar{x}, 2 \delta)$, (34) implies that $J_{x_{0}}(x) \in \mathbb{B}\left(\bar{y}, r_{\bar{y}}\right)$. In particular, let $x=\bar{x}$ in (33). Then, we obtain, from (33), that

$$
\left\|J_{x_{0}}(\bar{x})-\bar{y}\right\| \leq \varepsilon\left\|\bar{x}-x_{0}\right\|^{2}+\|\bar{y}\| .
$$


and therefore $J_{x_{0}}(\bar{x}) \in \mathbb{B}\left(\bar{y}, r_{\bar{y}}\right)$. Hence, by (32) and the Lipschitz-like property of $T_{\bar{x}}^{-1}$, we have

$$
\begin{aligned}
\operatorname{dist}\left(\bar{x}, \Phi_{x_{0}}(\bar{x})\right) & \leq M\left\|\bar{y}-J_{x_{0}}(\bar{x})\right\| \leq M \varepsilon\left\|x_{0}-\bar{x}\right\|^{2}+M\|\bar{y}\| \\
& =\left(1-\frac{22}{25}\right) r_{x_{0}}=c(1-r),
\end{aligned}
$$

which means that the assumption (2) of Lemma 2 is satisfied.

To fulfill assumption (3) of Lemma 2, let $x^{\prime}, x^{\prime \prime} \in \mathbb{B}\left(\bar{x}, r_{x_{0}}\right)$. Then for (30) and assumption (a), we have that $x^{\prime}, x^{\prime \prime} \in \mathbb{B}\left(\bar{x}, r_{\bar{x}}\right)$. Furthermore, for (33) we have that $J_{x_{0}}\left(x^{\prime}\right), J_{x_{0}}\left(x^{\prime \prime}\right) \in \mathbb{B}\left(\bar{y}, r_{\bar{y}}\right)$. This, together with Lipschitz-like property of $T_{\bar{x}}^{-1}$, implies that

$$
\begin{aligned}
e\left(\Phi_{x_{0}}\left(x^{\prime}\right)\right. & \left.\cap \mathbb{B}\left(\bar{x}, r_{x_{0}}\right), \Phi_{x_{0}}\left(x^{\prime \prime}\right)\right) \leq e\left(\Phi_{x_{0}}\left(x^{\prime}\right) \cap \mathbb{B}\left(\bar{x}, r_{\bar{x}}\right), \Phi_{x_{0}}\left(x^{\prime \prime}\right)\right) \\
& =e\left(T_{\bar{x}}^{-1}\left[J_{x_{0}}\left(x^{\prime}\right)\right] \cap \mathbb{B}\left(\bar{x}, r_{\bar{x}}\right), T_{\bar{x}}^{-1}\left[J_{x_{0}}\left(x^{\prime \prime}\right)\right]\right) \leq M\left\|J_{x_{0}}\left(x^{\prime}\right)-J_{x_{0}}\left(x^{\prime \prime}\right)\right\| .
\end{aligned}
$$

Applying (22) and continuous property of $\nabla^{2} f$, we get that

$$
\begin{aligned}
\left\|J_{x_{0}}\left(x^{\prime}\right)-J_{x_{0}}\left(x^{\prime \prime}\right)\right\| \leq & \left\|\nabla f(\bar{x})-\nabla f\left(x_{0}\right)\right\|\left\|x^{\prime}-x^{\prime \prime}\right\|+\frac{1}{2}\left\|\nabla^{2} f(\bar{x})-\nabla^{2} f\left(x_{0}\right)\right\|\left\|x^{\prime}-x^{\prime \prime}\right\|^{2} \\
& +\left\|\nabla^{2} f(\bar{x})\left(x^{\prime \prime}-\bar{x}\right)-\nabla^{2} f\left(x_{0}\right)\left(x^{\prime \prime}-x_{0}\right)\right\|\left\|x^{\prime}-x^{\prime \prime}\right\| \\
\leq & \varepsilon\left\|x^{\prime}-x^{\prime \prime}\right\|+\frac{1}{2} \varepsilon\left\|x^{\prime}-x^{\prime \prime}\right\|^{2}+\varepsilon\left\|x^{\prime}-x^{\prime \prime}\right\| \\
\leq & (2 \varepsilon+2 \varepsilon \delta)\left\|x^{\prime}-x^{\prime \prime}\right\| .
\end{aligned}
$$

Combining the above two inequalities we obtain that

$$
e\left(\Phi_{x_{0}}\left(x^{\prime}\right) \cap \mathbb{B}\left(\bar{x}, r_{x_{0}}\right), \Phi_{x_{0}}\left(x^{\prime \prime}\right)\right) \leq M(2 \varepsilon+2 \varepsilon \delta)\left\|x^{\prime}-x^{\prime \prime}\right\| .
$$

It follows, from (29), that

$$
e\left(\Phi_{x_{0}}\left(x^{\prime}\right) \cap \mathbb{B}\left(\bar{x}, r_{x_{0}}\right), \Phi_{x_{0}}\left(x^{\prime \prime}\right)\right) \leq \frac{22}{25}\left\|x^{\prime}-x^{\prime \prime}\right\|=r\left\|x^{\prime}-x^{\prime \prime}\right\| .
$$

This shows that the assumption (3) of Lemma 2 is also satisfied. Thus, by applying Lemma 2 we can deduce the existence of a fixed point $\hat{x}_{1} \in \mathbb{B}\left(\bar{x}, r_{x_{0}}\right)$ such that $\hat{x}_{1} \in \Phi_{x_{0}}\left(\hat{x}_{1}\right)$, which translates to $0 \in f\left(x_{0}\right)+\nabla f\left(x_{0}\right)\left(\hat{x}_{1}-x_{0}\right)+\frac{1}{2} \nabla^{2} f\left(x_{0}\right)\left(\hat{x}_{1}-x_{0}\right)^{2}+\mathcal{F}\left(\hat{x}_{1}\right)$ and so $\mathcal{D}\left(x_{0}\right) \neq \emptyset$. Accordingly, we can select $d_{0} \in \mathcal{D}\left(x_{0}\right)$ such that

$$
\left\|d_{0}\right\| \leq \eta \operatorname{dist}\left(0, \mathcal{D}\left(x_{0}\right)\right) .
$$

Then by Algorithm 2, $x_{1}:=x_{0}+d_{0}$ is defined.

Now we are ready to show that $(27)$ also holds for $n=0$. Since $\nabla^{2} f$ is continuous on $\mathbb{B}\left(\bar{x}, \frac{r_{\bar{x}}}{2}\right)$ with constant $\varepsilon$, we have the following inequality

$$
\varepsilon \geq \sup _{x \in \mathbb{B}\left(\bar{x}, \frac{r_{\bar{x}}}{2}\right)}\left\|\nabla^{2} f(x)-\nabla^{2} f(\bar{x})\right\| .
$$

Note that assumption (a) ensures $\alpha>0$. Therefore assumption (9) is satisfied by (10). The Lipschitz-like property of $T_{\bar{x}}^{-1}$ on $\mathbb{B}\left(\bar{y}, r_{\bar{y}}\right)$ relative to $\mathbb{B}\left(\bar{x}, r_{\bar{x}}\right)$ with constant $M$ implies, through Lemma 4 , that the mapping $T_{x}^{-1}$ is Lipschitz-like on $\mathbb{B}(\bar{y}, \alpha)$ relative to $\mathbb{B}\left(\bar{x}, \frac{r_{\bar{x}}}{2}\right)$ with constant $\frac{5 M}{2-5 M \varepsilon}$ for each $x \in \mathbb{B}\left(\bar{x}, \frac{r_{\bar{x}}}{2}\right)$. Specifically, for $x_{0} \in \mathbb{B}(\bar{x}, \hat{\delta}) \subseteq \mathbb{B}(\bar{x}, \delta) \subseteq \mathbb{B}\left(\bar{x}, \frac{r_{\bar{x}}}{2}\right)$ by assumption (a) and the choice of $\hat{\delta}$, we can say that $T_{x_{0}}^{-1}$ is Lipschitz-like on $\mathbb{B}(\bar{y}, \alpha)$ relative to $\mathbb{B}\left(\bar{x}, \frac{r_{\bar{x}}}{2}\right)$ with constant $\frac{5 M}{2-5 M \varepsilon}$. Furthermore, we can obtain, for (25) and (31), that

$$
\begin{aligned}
\operatorname{dist}\left(0, T_{x_{0}}\left(x_{0}\right)\right) & =\operatorname{dist}\left(0, f\left(x_{0}\right)+F\left(x_{0}\right)\right) \leq \varepsilon \delta^{2} \\
& \leq \frac{\alpha}{3} .
\end{aligned}
$$


As noted earlier that $x_{0} \in \mathbb{B}\left(\bar{x}, \frac{r_{\bar{x}}}{2}\right)$ and $0 \in \mathbb{B}\left(\bar{y}, \frac{\alpha}{3}\right)$ by (31)). This, together with (36), implies that Lemma 1 is applicable and hence by applying it, we have that

$$
\operatorname{dist}\left(x_{0}, T_{x_{0}}^{-1}(0)\right) \leq \frac{5 M}{2-5 M \varepsilon} \operatorname{dist}\left(0, T_{x_{0}}\left(x_{0}\right)\right) .
$$

This, together with (4), yields that

$$
\operatorname{dist}\left(0, \mathcal{D}\left(x_{0}\right)\right)=\operatorname{dist}\left(x_{0}, T_{x_{0}}^{-1}(0)\right) \leq \frac{5 M}{2-5 M \varepsilon} \operatorname{dist}\left(0, T_{x_{0}}\left(x_{0}\right)\right) .
$$

Because of (37), (31) and (23), (35) convert to the following inequality

$$
\begin{aligned}
\left\|x_{1}-x_{0}\right\| & =\left\|d_{0}\right\| \leq \eta \operatorname{dist}\left(0, \mathcal{D}\left(x_{0}\right)\right) \\
& \leq \frac{5 \eta M}{2-5 M \varepsilon} \operatorname{dist}\left(0, T_{x_{0}}\left(x_{0}\right)\right) \leq \frac{5 \eta M \varepsilon \delta^{2}}{2-5 M \varepsilon} \\
& =t\left(\frac{1}{10}\right) \delta .
\end{aligned}
$$

This shows that (27) holds for $n=0$.

We assume that $x_{1}, x_{2}, \ldots, x_{k}$ are obtained and (26) as well as $(27)$ are true for $n=0,1, \ldots, k-1$. We show that there exists $x_{k+1}$ such that assertions (26) and (27) hold for $n=k$. Since (26) and (27) are true for each $n \leq k-1$ and $t \leq 1$ by (24), we have the following inequality

$$
\begin{aligned}
\left\|x_{k}-\bar{x}\right\| & \leq \sum_{i=0}^{k-1}\left\|d_{i}\right\|+\left\|x_{0}-\bar{x}\right\| \leq \delta \sum_{i=0}^{k-1} t\left(\frac{1}{10}\right)^{2^{i}}+\delta \\
& \leq \delta \sum_{i=0}^{k-1}\left(\frac{1}{10}\right)^{2^{i}}+\delta \leq 2 \delta .
\end{aligned}
$$

This shows that (26) holds for $n=k$. Finally, we will show that the assertion (27) holds for $n=k$. Now with almost the same argument as we imposed for the case when $n=0$, we can prove that $\mathcal{D}\left(x_{k}\right) \neq \emptyset$, that is, the point $x_{k+1}$ exists and $T_{x_{k}}^{-1}$ is Lipschitz-like on $\mathbb{B}(\bar{y}, \alpha)$ relative to $\mathbb{B}\left(\bar{x}, \frac{r_{\bar{x}}}{2}\right)$ with constant $\frac{5 M}{2-5 M \varepsilon}$. Therefore, we have that

$$
\begin{aligned}
\left\|x_{k+1}-x_{k}\right\|= & \left\|d_{k}\right\| \leq \eta \operatorname{dist}\left(0, \mathcal{D}\left(x_{k}\right)\right) \leq \frac{5 \eta M}{2-5 M \varepsilon} \operatorname{dist}\left(0, T_{x_{k}}\left(x_{k}\right)\right) \\
= & \frac{5 \eta M}{2-5 M \varepsilon} \operatorname{dist}\left(0, f\left(x_{k}\right)+F\left(x_{k}\right)\right) \\
\leq & \frac{5 \eta M}{2-5 M \varepsilon} \| f\left(x_{k}\right)-f\left(x_{k-1}\right)-\nabla f\left(x_{k-1}\right)\left(x_{k}-x_{k-1}\right) \\
& -\frac{1}{2} \nabla^{2} f\left(x_{k-1}\right)\left(x_{k}-x_{k-1}\right)^{2} \| \\
\leq & \frac{5 \eta M \varepsilon}{2-5 M \varepsilon}\left\|x_{k}-x_{k-1}\right\|^{2} \leq \frac{5 \eta M \varepsilon \delta}{2-5 M \varepsilon}\left(t\left(\frac{1}{10}\right)^{2^{k-1}}\right)^{2} \delta \\
\leq & t\left(\frac{1}{10}\right)^{2^{k}} \delta
\end{aligned}
$$

This implies that (27) holds for $n=k$ and therefore the proof is completed.

Special case, when $\bar{x}$ is a solution of (1) i.e. $\bar{y}=0$, Theorem 1 is transformed to the following corollary, which provides the local convergent result for the extended cubic method. 
Corollary 1. Let $\bar{x}$ be a solution of $(1)$ and $\eta \in(1, \infty)$. Suppose that $T_{\bar{x}}^{-1}$ is pseudo-Lipschitz around $(0, \bar{x})$. Let $\beta>0$ and suppose that $\nabla^{2} f$ is continuous on $\mathbb{B}(\bar{x}, \beta)$ and that $f+\mathcal{F}$ is continuous at $\bar{x}$ for 0 , that $i s,(7)$ is true for $\bar{y}=0$. Then there exists some $\hat{\delta}>0$ such that any sequence $\left\{x_{n}\right\}$ generated by Algorithm 2 with starting point in $\mathbb{B}(\bar{x}, \hat{\delta})$ converges to a solution $x^{*}$ of (1).

Proof. By our assumption, $T_{\bar{x}}^{-1}$ is pseudo-Lipschitz around $(0, \bar{x})$. Then there exist constants $r_{0}, \beta$ and $M$ such that $T_{\bar{x}}^{-1}$ is Lipschitz-like on $\mathbb{B}\left(\bar{y}, r_{0}\right)$ relative to $\mathbb{B}(\bar{x}, \beta)$ with constant $M$. Then, for each $0<r \leq \beta$, one has that

$$
e\left(T_{\bar{x}}^{-1}\left(y_{1}\right) \cap \mathbb{B}(\bar{x}, r), T_{\bar{x}}^{-1}\left(y_{2}\right) \leq M\left\|y_{1}-y_{2}\right\| \quad \text { for any } y_{1}, y_{2} \in \mathbb{B}\left(0, r_{0}\right)\right.
$$

i.e. $T_{\bar{x}}^{-1}$ is Lipschitz-like on $\mathbb{B}\left(0, r_{0}\right)$ relative to $\mathbb{B}(\bar{x}, r)$ with constant $M$. Let $\varepsilon \in(0,1)$ be such that $M \varepsilon \leq \frac{2}{5}$. Then by the continuity of $\nabla^{2} f$ we can choose $r_{\bar{x}} \in(0, \beta)$ such that $\frac{r_{\bar{x}}}{2} \leq r, r_{0}-\frac{13}{4} \varepsilon r_{\bar{x}}^{2}>0$ and

$$
\varepsilon \geq \sup _{x, x^{\prime} \in \mathbb{B}\left(\bar{x}, \frac{r_{\bar{x}}}{2}\right)}\left\|\nabla^{2} f(x)-\nabla^{2} f\left(x^{\prime}\right)\right\|
$$

Then

$$
\alpha=\min \left\{r_{0}-\frac{13}{4} \varepsilon r_{\bar{x}}^{2}, \frac{r_{\bar{x}}(2-5 M \varepsilon)}{20 M}\right\}>0,
$$

and

$$
\min \left\{\frac{r_{\bar{x}}}{4}, \frac{\alpha}{3 \varepsilon}, \frac{r_{0}}{13 \varepsilon}\right\}>0
$$

Thus we can choose $0<\delta \leq 1$ such that

$$
\delta \leq \min \left\{\frac{r_{\bar{x}}}{4}, \frac{\alpha}{3 \varepsilon}, \frac{r_{0}}{13 \varepsilon}\right\}
$$

and

$$
5 M(1+10 \eta \delta) \varepsilon \leq 2 .
$$

Now it is one's duty to check that inequalities (a)-(c) of Theorem 1 hold. Thus, we can apply Theorem 1 to complete the proof of the corollary.

\subsection{Cubic Convergence}

This subsection is aimed to study the convergence analysis of the extended cubic method. In the following theorem we show that if $\nabla^{2} f$ is Lipschitz continuous around $\bar{x}$, then the sequence generated by Algorithm 2 is cubically convergent.

Theorem 2. Suppose that $T_{\bar{x}}^{-1}$ is Lipschitz-like on $\mathbb{B}\left(\bar{y}, r_{\bar{y}}\right)$ relative to $\mathbb{B}\left(\bar{x}, r_{\bar{x}}\right)$ with constant $M$ and that $\nabla^{2} f$ is Lipschitz continuous on $\mathbb{B}\left(\bar{x}, \frac{r_{\bar{x}}}{2}\right)$ with Lipschitz constant L. Let $\eta \in(1, \infty)$ and let

$$
\alpha:=\min \left\{r_{\bar{y}}-\frac{13}{4} L r_{\bar{x}}^{3}, \frac{r_{\bar{x}}\left(2-5 M L r_{\bar{x}}\right)}{20 M}\right\} .
$$

Let $\delta>0$ be such that

(a) $\delta \leq \min \left\{\frac{r_{\bar{x}}}{4}, \frac{125 r_{\bar{y}}}{76}, \frac{125 \alpha}{18}, 1\right\}$,

(b) $5(M+1) L\left(\eta \delta^{2}+r_{\bar{x}}\right) \leq 2$,

(c) $\|\bar{y}\|<\frac{L \delta^{3}}{6}$.

Suppose that $f+\mathcal{F}$ is continuous at $\bar{x}$ for $\bar{y}$, i.e. (7) holds. Then there exists some $\hat{\delta}>0$ such that any sequence $\left\{x_{n}\right\}$ generated by Algorithm 2 with initial point in $\mathbb{B}(\bar{x}, \hat{\delta})$ converges cubically to a solution $x^{*}$ of (1). 
Proof. According to the continuity of $f+\mathcal{F}$ at $\bar{x}$ for $\bar{y}$ and assumption (c), we can choose $0<\hat{\delta} \leq \delta$ be such that

$$
\operatorname{dist}\left(0, f\left(x_{0}\right)+F\left(x_{0}\right)\right) \leq \frac{L \delta^{3}}{6} \quad \text { for each } x_{0} \in \mathbb{B}(\bar{x}, \hat{\delta})
$$

Setting

$$
\gamma:=\frac{5 \eta M L \delta^{2}}{2-5 M L r_{\bar{x}}}
$$

It follows, from assumption (b), that

$$
\gamma \leq 1
$$

Let $x_{0} \in \mathbb{B}(\bar{x}, \hat{\delta})$. Analogous proof of Theorem 1, we use mathematical induction to show that Algorithm 2 generates at least one sequence and every sequence $\left\{x_{n}\right\}$ obtained by Algorithm 2 satisfies the following assertions:

$$
\begin{aligned}
& \Rightarrow x_{n}-\bar{x} \| \\
\text { and } \quad & \leq 2 \delta \\
\left\|d_{n}\right\| & \leq \gamma\left(\frac{1}{6}\right)^{3^{n}} \delta
\end{aligned}
$$

for each $n=0,1,2, \ldots$ Now, define

$$
r_{x}:=\frac{25}{96}\left(M L\|x-\bar{x}\|^{3}+6 M\|\bar{y}\|\right) \quad \text { for each } x \in X
$$

Because $\eta>1$ and $\delta \leq \frac{r_{\bar{x}}}{4}$ by assumption (a), it follows, from assumption (b), that

$$
\begin{aligned}
5(M+1) L \delta^{2} & =(M+1) L\left(\delta^{2}+4 \delta^{2}\right) \leq(M+1) L\left(\delta^{2}+4 \delta\right) \\
& \leq(M+1) L\left(\eta \delta^{2}+r_{\bar{x}}\right) \leq \frac{2}{5} .
\end{aligned}
$$

This gives

$$
M L \delta^{2} \leq \frac{2}{25} \quad \text { and } \quad L \delta^{2} \leq \frac{2}{25} .
$$

Thus, by $18 \delta \leq 125 \alpha$ in assumption (a) and second inequality in (44), we obtain that

$$
\|\bar{y}\|<\frac{L \delta^{3}}{6}=\frac{L \delta^{2}}{6} \cdot \delta \leq \frac{2}{25 \cdot 6} \cdot 25 \alpha=\frac{\alpha}{3}
$$

thanks to assumption (c). Thus, we obtain from (43), together with first inequality in (44) and assumption (c), that

$$
\begin{aligned}
r_{x} & <\frac{25}{96}\left(8 M L \delta^{3}+M L \delta^{3}\right) \\
& =\frac{25}{96}\left(9 M L \delta^{3}\right) \leq \frac{3}{16} \delta \leq 2 \delta \quad \text { for each } x \in \mathbb{B}(\bar{x}, 2 \delta) .
\end{aligned}
$$

It is clear that $\alpha>0$ by assumption (a). Then we have from (38) that

$$
\begin{aligned}
& \alpha>0 \Rightarrow 2-5 M L r_{\bar{x}}>0 \Rightarrow 5 M L r_{\bar{x}}<2 \\
\Rightarrow & 20 M L \delta<5 M L r_{\bar{x}}<2 \Rightarrow L M \delta<\frac{1}{10} .
\end{aligned}
$$

Note that, for $n=0,(41)$ is trivial. To show that the point $x_{1}$ exists and (42) holds for $n=0$, it suffices to prove that $\mathcal{D}\left(x_{0}\right) \neq \emptyset$. We will do that by applying Lemma 2 to the mapping $\Phi:=\Phi_{x_{0}}$. To do this, let us check that both assumptions (2) and (3) of Lemma 2 hold with $c:=r_{x_{0}}$ and $r:=\frac{9}{25}$. Here, we note that $\bar{x} \in T_{\bar{x}}^{-1}(\bar{y}) \cap \mathbb{B}(\bar{x}, \delta)$. Then by the definition of the excess $e$, we obtain that

$$
\begin{aligned}
\operatorname{dist}\left(\bar{x}, \Phi_{x_{0}}(\bar{x})\right) & \leq e\left(T_{\bar{x}}^{-1}(\bar{y}) \cap \mathbb{B}(\bar{x}, \delta), \Phi_{x_{0}}(\bar{x})\right) \\
& \leq e\left(T_{\bar{x}}^{-1}(\bar{y}) \cap \mathbb{B}\left(\bar{x}, r_{\bar{x}}\right), T_{\bar{x}}^{-1}\left[J_{x_{0}}(\bar{x})\right]\right) .
\end{aligned}
$$


By the Lipschitz continuity property of $\nabla^{2} f$, we obtain, for each $x \in \mathbb{B}(\bar{x}, 2 \delta) \subseteq \mathbb{B}\left(\bar{x}, \frac{r_{\bar{x}}}{2}\right)$, that

$$
\begin{aligned}
\left\|J_{x_{0}}(x)-\bar{y}\right\|= & \| f(\bar{x})+\nabla f(\bar{x})(x-\bar{x})+\frac{1}{2} \nabla^{2} f(\bar{x})(x-\bar{x})^{2}-f\left(x_{0}\right)-\nabla f\left(x_{0}\right)\left(x-x_{0}\right) \\
& -\frac{1}{2} \nabla^{2} f\left(x_{0}\right)\left(x-x_{0}\right)^{2}-\bar{y} \| \\
\leq & \left\|f(x)-f\left(x_{0}\right)-\nabla f\left(x_{0}\right)\left(x-x_{0}\right)-\frac{1}{2} \nabla^{2} f\left(x_{0}\right)\left(x-x_{0}\right)^{2}\right\| \\
& +\left\|f(x)-f(\bar{x})-\nabla f(\bar{x})(x-\bar{x})+\frac{1}{2} \nabla^{2} f(\bar{x})(x-\bar{x})^{2}\right\|+\|\bar{y}\| \\
\leq & \frac{L}{6}\left(\left\|x-x_{0}\right\|^{3}+\|x-\bar{x}\|^{3}\right)+\|\bar{y}\| .
\end{aligned}
$$

Because of $\left\|x_{0}-\bar{x}\right\| \leq \hat{\delta} \leq \delta, 76 \delta \leq 125 r_{\bar{y}}$ by assumption (a), $\|\bar{y}\|<\frac{L \delta^{3}}{6}$ by assumption (c) and second relation in (44), (49) implies that

$$
\begin{aligned}
\left\|J_{x_{0}}(x)-\bar{y}\right\| & \leq \frac{L}{6}\left(\left\|(x-\bar{x})+\left(\bar{x}-x_{0}\right)\right\|^{3}+\|x-\bar{x}\|^{3}\right)+\frac{L \delta^{3}}{6} \\
& \leq \frac{L}{6}\left((3 \delta)^{3}+(2 \delta)^{3}\right)+\frac{L \delta^{3}}{6}=6 L \delta^{3} \\
& =6 \cdot L \delta^{2} \cdot \delta \leq r_{\bar{y}} .
\end{aligned}
$$

This means that, for each $x \in \mathbb{B}(\bar{x}, 2 \delta), J_{x_{0}}(x) \in \mathbb{B}\left(\bar{y}, r_{\bar{y}}\right)$. Specifically, putting $x=\bar{x}$ in (49). Then we have that

$$
\begin{aligned}
\left\|J_{x_{0}}(\bar{x})-\bar{y}\right\| & \leq \frac{L}{6}\left\|\bar{x}-x_{0}\right\|^{3}+\|\bar{y}\| \\
& \leq \frac{L \delta^{3}}{6}+\frac{L \delta^{3}}{6} \leq r_{\bar{y}} .
\end{aligned}
$$

Hence, by (51) and the Lipschitz-like property of $T_{\bar{x}}^{-1}$, we have, from (48), that

$$
\begin{aligned}
\operatorname{dist}\left(\bar{x}, \Phi_{x_{0}}(\bar{x})\right) & \leq M\left\|\bar{y}-J_{x_{0}}(\bar{x})\right\| \leq \frac{M L}{6}\left\|\bar{x}-x_{0}\right\|^{3}+M\|\bar{y}\| \\
& =\left(1-\frac{9}{25}\right) r_{x_{0}}=c(1-r),
\end{aligned}
$$

which shows that the assumption (2) of Lemma 2 is satisfied.

Next, we show that assumption (3) of Lemma 2 is satisfied. To do this, let $x^{\prime}, x^{\prime \prime} \in \mathbb{B}\left(\bar{x}, r_{x_{0}}\right)$. Then we have that $x^{\prime}, x^{\prime \prime} \in \mathbb{B}\left(\bar{x}, r_{x_{0}}\right) \subseteq \mathbb{B}(\bar{x}, 2 \delta) \subseteq \mathbb{B}\left(\bar{x}, r_{\bar{x}}\right)$ by $(46)$ and $J_{x_{0}}\left(x^{\prime}\right), J_{x_{0}}\left(x^{\prime \prime}\right) \in \mathbb{B}\left(\bar{y}, r_{\bar{y}}\right)$ by (50). This, together with the Lipschitz-like property of $T_{\bar{x}}^{-1}$, implies that

$$
\begin{aligned}
e\left(\Phi_{x_{0}}\left(x^{\prime}\right) \cap \mathbb{B}\left(\bar{x}, r_{x_{0}}\right), \Phi_{x_{0}}\left(x^{\prime \prime}\right)\right) & \leq e\left(\Phi_{x_{0}}\left(x^{\prime}\right) \cap \mathbb{B}\left(\bar{x}, r_{\bar{x}}\right), \Phi_{x_{0}}\left(x^{\prime \prime}\right)\right) \\
& =e\left(T_{\bar{x}}^{-1}\left[J_{x_{0}}\left(x^{\prime}\right)\right] \cap \mathbb{B}\left(\bar{x}, r_{\bar{x}}\right), T_{\bar{x}}^{-1}\left[J_{x_{0}}\left(x^{\prime \prime}\right)\right]\right) \\
& \leq M\left\|J_{x_{0}}\left(x^{\prime}\right)-J_{x_{0}}\left(x^{\prime \prime}\right)\right\| .
\end{aligned}
$$

Since $\nabla^{2} f$ and $\nabla^{2} f(\cdot)(z-\cdot)$ are Lipschitz continuous on $\mathbb{B}\left(\bar{x}, \frac{r_{\bar{x}}}{2}\right)$ for all $z \in \mathbb{B}\left(\bar{x}, \frac{r_{\bar{x}}}{2}\right)$, then $\nabla f$ is also Lipschitz continuous on $\mathbb{B}\left(\bar{x}, \frac{r_{\bar{x}}}{2}\right)$ and for simplicity we take the same Lipschitz constant $L$. Thus, for the choice of $x_{0},(22)$ yields that

$$
\begin{aligned}
\left\|J_{x_{0}}\left(x^{\prime}\right)-J_{x_{0}}\left(x^{\prime \prime}\right)\right\| \leq & \left\|\nabla f(\bar{x})-\nabla f\left(x_{0}\right)\right\|\left\|x^{\prime}-x^{\prime \prime}\right\|+\frac{1}{2}\left\|\nabla^{2} f(\bar{x})-\nabla^{2} f\left(x_{0}\right)\right\|\left\|x^{\prime}-x^{\prime \prime}\right\|^{2} \\
& +\left\|\nabla^{2} f(\bar{x})\left(x^{\prime \prime}-\bar{x}\right)-\nabla^{2} f\left(x_{0}\right)\left(x^{\prime \prime}-x_{0}\right)\right\|\left\|x^{\prime}-x^{\prime \prime}\right\| \\
\leq & L\left\|\bar{x}-x_{0}\right\|\left\|x^{\prime}-x^{\prime \prime}\right\|+\frac{L}{2}\left\|\bar{x}-x_{0}\right\|\left\|x^{\prime}-x^{\prime \prime}\right\|^{2}+L\left\|\bar{x}-x_{0}\right\|\left\|x^{\prime}-x^{\prime \prime}\right\| \\
\leq & \left(2 L+\frac{L}{2}\left\|x^{\prime}-x^{\prime \prime}\right\|\right)\left\|\bar{x}-x_{0}\right\|\left\|x^{\prime}-x^{\prime \prime}\right\| \\
\leq & 2 L\left(\delta+\delta^{2}\right)\left\|x^{\prime}-x^{\prime \prime}\right\| .
\end{aligned}
$$


It follows, from (47) and first inequality of (44), that

$$
\begin{aligned}
e\left(\Phi_{x_{0}}\left(x^{\prime}\right) \cap \mathbb{B}\left(\bar{x}, r_{x_{0}}\right), \Phi_{x_{0}}\left(x^{\prime \prime}\right)\right) & \leq\left(2 M L \delta+2 M L \delta^{2}\right)\left\|x^{\prime}-x^{\prime \prime}\right\| \\
& \leq \frac{9}{25}\left\|x^{\prime}-x^{\prime \prime}\right\|=r\left\|x^{\prime}-x^{\prime \prime}\right\| .
\end{aligned}
$$

This means that the assumption (3) of Lemma 2 is also satisfied. Thus by Lemma 2, we can deduce the existence of a fixed point $\hat{x}_{1} \in \mathbb{B}\left(\bar{x}, r_{x_{0}}\right)$ such that $\hat{x}_{1} \in \Phi_{x_{0}}\left(\hat{x}_{1}\right)$, which translates to $0 \in f\left(x_{0}\right)+$ $\nabla f\left(x_{0}\right)\left(\hat{x}_{1}-x_{0}\right)+\frac{1}{2} \nabla^{2} f\left(x_{0}\right)\left(\hat{x}_{1}-x_{0}\right)^{2}+\mathcal{F}\left(\hat{x}_{1}\right)$ and hence $\mathcal{D}\left(x_{0}\right) \neq \emptyset$. Consequently, we can choose $d_{0} \in \mathcal{D}\left(x_{0}\right)$ such that

$$
\left\|d_{0}\right\| \leq \eta \operatorname{dist}\left(0, \mathcal{D}\left(x_{0}\right)\right)
$$

Therefore, according to the Algorithm 2, we can say that $x_{1}:=x_{0}+d_{0}$ is defined.

Below we show that assertion (42) also holds for $n=0$. Since $\nabla^{2} f$ is Lipschitz continuous on $\mathbb{B}\left(\bar{x}, \frac{r_{\bar{x}}}{2}\right)$ with Lipschitz constant $L$, we have, for $x^{\prime}, x^{\prime \prime} \in \mathbb{B}\left(\bar{x}, \frac{r_{\bar{x}}}{2}\right)$, that

$$
\begin{aligned}
L r_{\bar{x}} & \geq L\left\|x^{\prime}-x^{\prime \prime}\right\| \geq\left\|\nabla^{2} f\left(x^{\prime}\right)-\nabla^{2} f\left(x^{\prime \prime}\right)\right\| \\
& \geq \sup _{x \in \mathbb{B}\left(\bar{x}, \frac{r_{\bar{x}}}{2}\right)}\left\|\nabla^{2} f(x)-\nabla^{2} f(\bar{x})\right\| .
\end{aligned}
$$

Since $\alpha>0$ by assumption (a), (10) and (53) imply that assumption (9) is satisfied with $\varepsilon:=L r_{\bar{x}}$. Note by assumption (a) that $x_{0} \in \mathbb{B}(\bar{x}, \hat{\delta}) \subseteq \mathbb{B}(\bar{x}, \delta) \subseteq \mathbb{B}\left(\bar{x}, \frac{r_{\bar{x}}}{2}\right)$. Therefore, since $T_{\bar{x}}^{-1}$ is Lipschitz-like on $\mathbb{B}\left(\bar{y}, r_{\bar{y}}\right)$ relative to $\mathbb{B}\left(\bar{x}, r_{\bar{x}}\right)$, it follows from Lemma 4 that $T_{x_{0}}^{-1}$ is Lipschitz-like on $\mathbb{B}(\bar{y}, \alpha)$ relative to $\mathbb{B}\left(\bar{x}, \frac{r_{\bar{x}}}{2}\right)$ with constant $\frac{5 M}{2-5 M L r_{\bar{x}}}$. Moreover, (39) and (45) imply that

$$
\begin{aligned}
\operatorname{dist}\left(0, T_{x_{0}}\left(x_{0}\right)\right) & =\operatorname{dist}\left(0, f\left(x_{0}\right)+F\left(x_{0}\right)\right) \leq \frac{L \delta^{3}}{6} \\
& \leq \frac{\alpha}{3}
\end{aligned}
$$

It has been mentioned above that $x_{0} \in \mathbb{B}\left(\bar{x}, \frac{r_{\bar{x}}}{2}\right)$ and by $\left.(45)\right)$ we have that $0 \in \mathbb{B}\left(\bar{y}, \frac{\alpha}{3}\right)$. This, together with (54), implies that Lemma 1 is applicable and hence by applying it we have that

$$
\operatorname{dist}\left(x_{0}, T_{x_{0}}{ }^{-1}(0)\right) \leq \frac{5 M}{2-5 M L r_{\bar{x}}} \operatorname{dist}\left(0, T_{x_{0}}\left(x_{0}\right)\right)
$$

Applying (55), we have from (4) that

$$
\begin{aligned}
\operatorname{dist}\left(0, \mathcal{D}\left(x_{0}\right)\right) & =\operatorname{dist}\left(x_{0}, T_{x_{0}}^{-1}(0)\right) \\
& \leq \frac{5 M}{2-5 M L r_{\bar{x}}} \operatorname{dist}\left(0, T_{x_{0}}\left(x_{0}\right)\right) .
\end{aligned}
$$

Utilizing (56), (54) and then (40) in (52), we obtain that

$$
\begin{aligned}
\left\|x_{1}-x_{0}\right\| & =\left\|d_{0}\right\| \leq \eta \operatorname{dist}\left(0, \mathcal{D}\left(x_{0}\right)\right) \\
& \leq \frac{5 \eta M}{2-5 M L r_{\bar{x}}} \operatorname{dist}\left(0, T_{x_{0}}\left(x_{0}\right)\right) \leq \frac{5 \eta M L \delta^{3}}{6\left(2-5 M L r_{\bar{x}}\right)} \\
& =\gamma\left(\frac{1}{6}\right) \delta .
\end{aligned}
$$

This shows that (42) holds for $n=0$.

We assume that the points $x_{1}, x_{2}, \ldots, x_{k}$ are generated by Algorithm 2, and (41) and (42) are true for $n=0,1, \ldots, k-1$. We show that there exists $x_{k+1}$ such that (41) and (42) hold for $n=k$. Since, for 
each $n \leq k-1,(41)$ and (42) are true and $\gamma \leq 1$ by (40), we have the following inequality

$$
\begin{aligned}
\left\|x_{k}-\bar{x}\right\| & \leq \sum_{i=0}^{k-1}\left\|d_{i}\right\|+\left\|x_{0}-\bar{x}\right\| \leq \delta \sum_{i=0}^{k-1} \gamma\left(\frac{1}{6}\right)^{3^{i}}+\delta \\
& \leq \delta \sum_{i=0}^{k-1}\left(\frac{1}{6}\right)^{3^{i}}+\delta \leq 2 \delta .
\end{aligned}
$$

This shows that (41) holds for $n=k$. Finally, we will show that (42) holds for $n=k$. Now with almost analogous arguments that we used for the case when $n=0$, we can prove that $\mathcal{D}\left(x_{k}\right) \neq \emptyset$ and so by Algorithm 2 we can choose $d_{k} \in \mathcal{D}\left(0, x_{k}\right)$ such that

$$
\left\|d_{k}\right\| \leq \eta \operatorname{dist}\left(0, \mathcal{D}\left(x_{k}\right)\right),
$$

that is, the point $x_{k+1}$ exists. Moreover, we have that $T_{x_{k}}^{-1}$ is Lipschitz-like on $\mathbb{B}(\bar{y}, \alpha)$ relative to $\mathbb{B}\left(\bar{x}, \frac{r_{\bar{x}}}{2}\right)$ with constant $\frac{5 M}{2-5 M L r_{\bar{x}}}$. Therefore, we have that

$$
\begin{aligned}
\left\|x_{k+1}-x_{k}\right\| & =\left\|d_{k}\right\| \leq \eta \operatorname{dist}\left(0, \mathcal{D}\left(x_{k}\right)\right) \\
& \leq \frac{5 \eta M}{2-5 M L r_{\bar{x}}} \operatorname{dist}\left(0, T_{x_{k}}\left(x_{k}\right)\right)=\frac{5 \eta M}{2-5 M L r_{\bar{x}}} \operatorname{dist}\left(0, f\left(x_{k}\right)+F\left(x_{k}\right)\right) \\
& \leq \frac{5 \eta M}{2-5 M L r_{\bar{x}}}\left\|f\left(x_{k}\right)-f\left(x_{k-1}\right)-\nabla f\left(x_{k-1}\right)\left(x_{k}-x_{k-1}\right)-\frac{1}{2} \nabla^{2} f\left(x_{k-1}\right)\left(x_{k}-x_{k-1}\right)^{2}\right\| \\
& \leq \frac{5 \eta M L}{6\left(2-5 M L r_{\bar{x}}\right)}\left\|x_{k}-x_{k-1}\right\|^{3} \leq \frac{5 \eta M L \delta^{2}}{6\left(2-5 M L r_{\bar{x}}\right)}\left(\gamma\left(\frac{1}{6}\right)^{3^{k-1}}\right)^{3} \delta \\
& \leq \gamma\left(\frac{1}{6}\right)^{3^{k}} \delta .
\end{aligned}
$$

This implies that (42) holds for $n=k$ and therefore the proof of the theorem is completed.

In the special case when $\bar{x}$ is a solution of (1) (that is $\bar{y}=0$ in Theorem 2), then we have the following corollary which gives the cubically local convergence result for the extended cubic method. The proof of this corollary is similar to that we have done for Corollary 1.

Corollary 2. Suppose that $\eta>1$ and that $\bar{x}$ is a solution of (1) and that $T_{\bar{x}}^{-1}$ is pseudo-Lipschitz around $(0, \bar{x})$. Suppose that $\nabla^{2} f$ is Lipschitz continuous around $\bar{x}$. Suppose that

$$
\lim _{x \rightarrow \bar{x}} \operatorname{dist}(0, f(x)+\mathcal{F}(x))=0 .
$$

Then there exists some $\hat{\delta}>0$ such that any sequence $\left\{x_{n}\right\}$ generated by Algorithm 2 with initial point in $\mathbb{B}(\bar{x}, \hat{\delta})$ converges cubically to a solution $x^{*}$ of $(1)$.

\section{Concluding Remarks}

The semilocal and local convergence results for the extended cubic method are established when $\eta>1$ and $T_{\bar{x}}^{-1}$ is Lipschitz-like as well as $\nabla^{2} f$ is continuous or Lipschitz continuous. The results seem new for the generalized equation problem (1). Though the results are true for the extended cubic method, this method is little complicated for the case when $\eta=1$. However, from the proofs of the main theorems, one sees that all results obtained in the present paper remain true provided the following implication holds for each $x \in X$ :

$$
\mathcal{D}(x) \neq \emptyset \Longrightarrow \exists \bar{d} \in \mathcal{D}(x) \text { such that }\|\bar{d}\|=\min _{d \in \mathcal{D}(x)}\|d\| .
$$

To see the detail proof of the above implication, one can refer to [10]. 


\section{Acknowledgments}

The authors thank the referees and the associate editor for their valuable comments and constructive suggestions which improved the presentation of this manuscript. This work is fully supported by Ministry of Science and Technology, Bangladesh, Grant No. 39.009.002.01.00.057.2015-2016/EAS-326.

\section{References}

1. S. M. Robinson, "Generalized equations and their solutions, part I: basic theory", Math. Progamming Stud., vol. 10, pp. 128-141, 1979 .

2. S. M. Robinson, "Generalized equations and their solutions, part II: applications to nonlinear programming",Math. Programming Stud., vol. 19, pp. 200-221, 1982.

3. S. M. Robinson, "Strong regular generalized equations", Math. of Oper. Res., vol. 5, pp. 43-62, 1980.

4. A. L. Dontchev, Local convergence of the Newton method for generalized equation, C. R. A. S Paris Ser.I, vol. 322, pp. 327-331, 1996.

5. A. L. Dontchev, "Uniform convergence of the Newton method for Aubin continuous maps", Serdica Math. J., vol. 22, pp. 385-398, 1996.

6. A. Piétrus, "Generalized equations under mild differentiability conditions", Rev. R. Acad. Cienc. Exact. Fis. Nat., vol. 94, no. 1, pp. 15-18, 2000.

7. A. Piétrus, "Does Newton's method for set-valued maps converges uniformly in mild differentiability context?", Rev. Colombiana Mat., vol. 34, pp. 49-56, 2000.

8. A. L. Dontchev, "Local analysis of a Newton-type method based on partial linearization", Lectures in Applied Mathematics, vol. 32, pp. 295-306, 1996.

9. M. H. Geoffroy, S. Hilout and A. Piétrus, "Acceleration of convergence in Dontchev's iterative method for solving variational inclusions", Serdica Math. J., vol. 29, pp. 45-54, 2003.

10. M. H. Rashid, S. H. Yu, C. Li and S. Y. Wu, "Convergence analysis of the Gauss-Newton-type method for Lipschitz-like mappings", J. Optim. Theory Appl., vol. 158, no. 1, pp. 216-233, 2013.

11. C. Li, W. H. Zhang and X. Q. Jin, "Convergence and uniqueness properties of Gauss-Newton's method", Comput. Math. Appl., vol. 47, pp. 1057-1067, 2004.

12. J. P. Dedieu and M. H. Kim, "Newton's method for analytic systems of equations with constant rank derivatives", J. Complexity, vol. 18, pp. 187-209, 2002.

13. J. P. Dedieu and M. Shub, "Newton's method for overdetermined systems of equations", Math. Comp., vol. 69, pp. 1099-1115, 2000.

14. J. S. He, J. H. Wang and C. Li, "Newton's method for underdetemined systems of equations under the modified $\gamma$-condition", Numer. Funct. Anal. Optim., vol. 28, pp. 663-679, 2007.

15. X. B. Xu, and C. Li, "Convergence of Newton's method for systems of equations with constant rank derivatives",J. Comput. Math., vol. 25, pp. 705-718, 2007.

16. X. B. Xu, and C. Li, "Convergence criterion of Newton's method for singular systems with constant rank derivatives", J. Math. Anal. Appl., vol. 345, pp. 689-701, 2008.

17. M. H. Rashid, "Convergence Analysis of a Variant of Newton-type Method for Generalized Equations", International Journal of Computer Mathematics, vol. 95, issue 3, pp. 584-600, 2018.

18. M. H. Rashid, "On the convergence of extended Newton-type method for solving variational inclusions", Cogent Mathematics, vol. 1, no. 1, 2014. DOI 10.1080/23311835.2014.980600

19. J. P. Aubin, "Lipschitz behavior of solutions to convex minimization problems", Math. Oper. Res., vol. 9, pp. $87-111,1984$

20. J. P. Aubin and H. Frankowska, Set-valued Analysis, Birkhäuser, Boston, 1990.

21. A. L. Dontchev and W. W. Hager, "An inverse mapping theorem for set-valued maps", Proc. Amer. Math. Soc., vol. 121, pp. 481-489, 1994.

22. M. H. Geoffroy, S. Hilout and A. Piétrus, "Stability of a cubically convergent method for generalized equations", Set-Valued Analysis, vol. 14, pp. 41-54, 2006.

23. M. H. Rashid, "Convergence Analysis of Extended Hummel-Seebeck-type Method for Solving Variational Inclusions", Vietnam Journal of Mathematics, vol. 44, no. 1, pp. 1-14, 2015. 